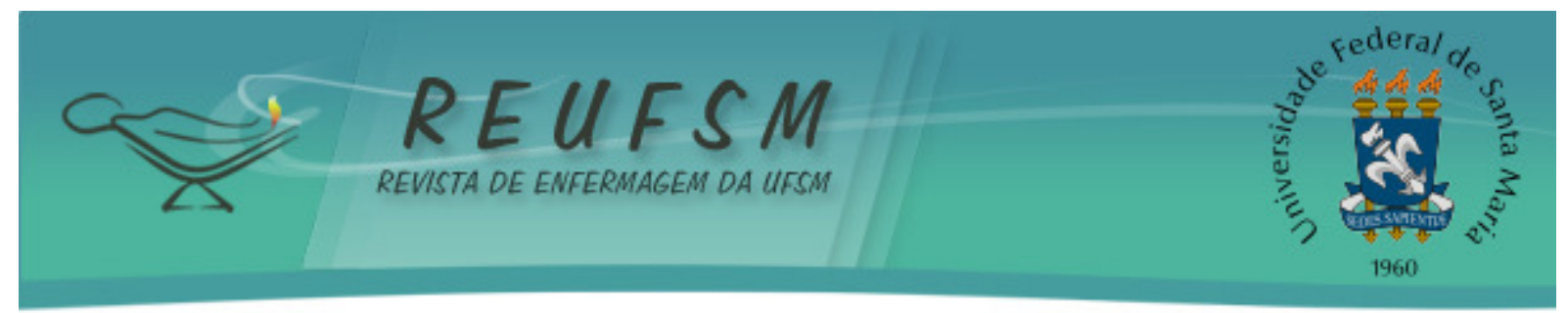

\title{
ARTIGO ORIGINAL CONHECIMENTO DE MULHERES SOBRE FATORES RELACIONADOS AO CÂNCER DE MAMA
}

AWARENESS OF WOMEN REGARDING BREAST CANCER

\section{CONOCIMIENTO DE LAS MUJERES SOBRE LOS FACTORES RELACIONADOS AL CÁNCER DE MAMA}

Doi: 10.5902/2179769225641

\author{
Neide Derenzo ${ }^{1}$ \\ Maria Antonia Ramos Costa ${ }^{2}$ \\ Willian Augusto de Melo ${ }^{3}$ \\ Cassia Kely Favoretto Costa ${ }^{4}$ \\ Verônica Francisqueti ${ }^{5}$ \\ Marcelo Picinin Bernuci ${ }^{6}$
}

RESUMO: Objetivo: descrever o perfil e conhecimento de mulheres atendidas pela Estratégia Saúde da Família sobre fatores relacionados ao câncer de mama. Método: trata-se de um estudo descritivo, transversal e quantitativo, desenvolvido em município do noroeste Paranaense onde participaram, por amostragem aleatória, 193 mulheres. A coleta de dados ocorreu de agosto de 2014 a outubro de 2015, por meio de entrevista e foram analisados por meio de estatística descritiva, verificando-se medidas de proporções simples e de tendência central. Resultados: prevaleceram mulheres entre 50-60 anos, com baixa escolaridade, maioria casadas e etnia branca. Negaram história familiar de câncer $81 \%$, porém $52,1 \%$ consideraram como maior fator de risco, $82,3 \%$ utilizaram anticoncepcionais. Conclusão: Observou que a maioria apreendia conhecimentos sobre os fatores de risco para o desenvolvimento do câncer de mama, respectivamente nos quesitos história familiar, estilo de vida e história reprodutiva.

Descritores: Neoplasias da mama; Saúde da mulher; Conhecimentos, Atitudes e práticas em saúde; Prevenção primária.

ABSTRACT: Aim: to describe the profile and awareness of women assisted by the Family Health Strategy on factors related to breast cancer. Method: this is a descriptive, crosssectional and quantitative study, developed in a municipality in northwest Parana, where 193 women participated randomly. The data were collected from August 2014 to October 2015, through an interview and analyzed by means of descriptive statistics, verifying measures of

\footnotetext{
${ }^{1}$ Enfermeira, Mestre em Promoção em Saúde. Docente do Curso de Enfermagem da Universidade Estadual do Paraná (UNESPAR), Paranavaí, Paraná, Brasil. E-mail: neidederenzo@ hotmail.com

${ }^{2}$ Enfermeira, Doutora em Enfermagem. Docente do Curso de Enfermagem da Universidade Estadual do Paraná (UNESPAR), Paranavaí, Paraná, Brasil. E-mail: enfunespar@hotmail.com

${ }^{3}$ Enfermeiro, Doutor em Ciências da Saúde. Docente do Curso de Enfermagem da Universidade Estadual do Paraná (UNESPAR), Paranavaí, Paraná, Brasil. E-mail: profewill@ yahoo.com.br

${ }^{4}$ Economista. Doutora em Economia Aplicada. Docente da Universidade Estadual de Maringá (UEM), Maringá,Parana', Brasil. E -mail: ckfcosta@uem.br

${ }^{5}$ Graduanda do Curso de Enfermagem. Universidade Estadual do Paraná (UNESPAR), Paranavaí, Paraná, Brasil. E mail: veronicafrancisqueti@hotmail.com

${ }^{6}$ Biólogo. Pós-doutorado no Departamento de Ginecologia e Obstetrícia. Docente do Programa de Pósgraduação em Promoção da Saúde e do Curso de Medicina do Centro Universitário (UNICESUMAR), Maringá, Parana, Brasil. E-mail: marcelo.bernuci@unicesumar.edu.br
} 


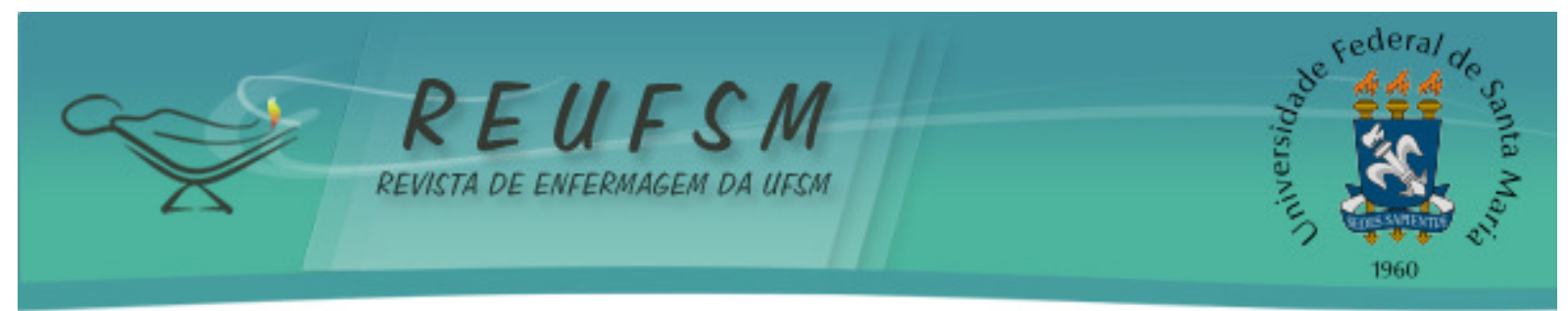

simple proportions and central tendency. Results: women between 50-60 years old, with low schooling, mostly married and white, prevailed. Eighty-one percent of the women denied a family history of cancer, but $52.1 \%$ considered it to be a major risk factor and $82.3 \%$ used contraceptives. Conclusion: it was observed that the majority apprehended knowledge about the risk factors for the development of breast cancer, respectively in the questions family history, lifestyle and reproductive history.

Descriptors: Breast neoplasms; Women's health; Health knowledge, attitudes, practice; Primary prevention.

RESUMEN: Objetivo: describir el perfil y el conocimiento de las mujeres, atendidas por la Estrategia Salud de la Familia, sobre factores relacionados al cáncer de mama. Método: se trata de un estudio descriptivo, transversal y cuantitativo, desarrollado en municipio del noroeste Paranaense en el cual participaron, por muestreo aleatorio, 193 mujeres. La recolección de datos ocurrió de agosto de 2014 a octubre de 2015, por medio de entrevista y fueron analizados con base en estadística descriptiva, verificándose medidas de proporciones simples y de tendencia central. Resultados: prevalecieron mujeres entre 50-60 años, con baja escolaridad, mayoría casadas y etnia blanca. Negaron historia familiar de cáncer $81 \%$, pero $52,1 \%$ consideraron como mayor factor de riesgo, 82,3\% utilizaron anticonceptivos. Conclusión: Observó que la mayoría aprehendía conocimientos sobre los factores de riesgo para el desarrollo del cáncer de mama, respectivamente en los antecedentes familiares, estilo de vida e historia reproductiva.

Descriptores: Neoplasias de la mama; Salud de la mujer; Conocimientos, actitudes y práctica en salud; Prevención primaria.

\section{INTRODUÇÃO}

O câncer de mama tem sido considerado um grave problema de saúde pública, pois se configura como a causa mais comum de morte entre as mulheres. ${ }^{1}$ Embora a incidência deste tipo de câncer tenha aumentado expressivamente ao longo das últimas décadas e esteja positivamente associada com baixos índices de desenvolvimento humano (IDH), a taxa de mortalidade sofre progressiva regressão em países de alta renda. $^{2-3}$ Verifica-se de maneira contrastantes e preocupantes as crescentes taxas de incidência e mortalidade em vários países, particularmente aqueles submetidos a mudanças rápidas no índice de desenvolvimento humano. ${ }^{2-3}$

Estudos recentes apontam que os incrementos na incidência do câncer de mama podem estar relacionados ao aumento da expectativa de vida, ao processo de urbanização e de industrialização bem como à melhora da tecnologia diagnóstica e a ampliação de programas de rastreamento. ${ }^{4-6}$ No entanto, tendências de aumento de incidência e morte refletem diferenças nos padrões de fatores de risco, acesso e disponibilidade à detecção precoce e tratamento oportuno. Relatos da associação entre altos índices de mortalidade, diagnóstico 


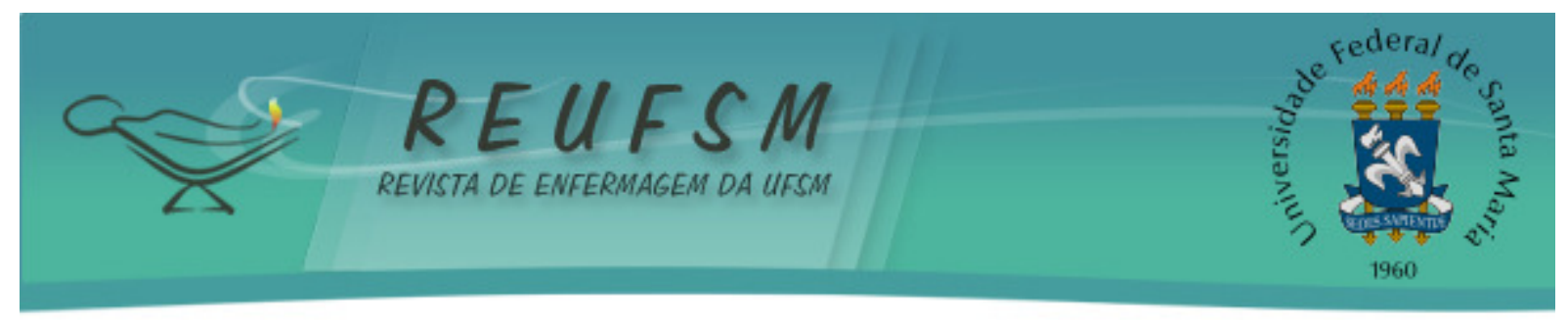

tardio e assistência médica incipiente, têm sido amplamente divulgados e parecem ser frequentes nos países em desenvolvimento. ${ }^{7-9}$

Este cenário hodierno se reflete no Brasil, onde a estimativa de novos casos de câncer de mama se assemelha a países de baixo desenvolvimento. ${ }^{10}$ Apenas para o ano de 2015 foi estimado o risco de 56,09 casos a cada 100 mil mulheres, e na região sudeste, uma das áreas mais desenvolvidas do país, embora o número de mortes por esta neoplasia tenha se estabilizado, a alta incidência ainda é um fator preocupante. ${ }^{1,11}$ Para o Estado do Paraná, a taxa de incidência em 2014 foi de 61,76 casos a cada 100 mil mulheres, e o número de óbitos no período compreendido entre os anos 2000 a 2012 ultrapassou 8.000 mulheres. ${ }^{12}$ Este perfil não se difere nos municípios com alto índice de IDH, como é o caso do município de Paranavaí, localizado na região Noroeste do Paraná, sul do Brasil, que apresenta aumento crescente de mortalidade por esta neoplasia maligna, passando de seis mortes em 2013 para dez em 2014.

Virtuosamente, em países desenvolvidos, os índices de mortalidade foram reduzidos de maneira expressiva após maciço investimento e organização do rastreamento e diagnóstico precoce organizado. ${ }^{13}$ No entanto, no Brasil, o rastreamento oportunístico prevalece, no qual a população procura de maneira espontânea as unidades de saúde para investigação. ${ }^{1}$

Embora existam estratégias e programas para orientar e estimular a prevenção ao câncer de mama, a eficácia dessas medidas é questionável. A adesão aos métodos preventivos ainda é limitada, visto que grande parte das mulheres busca ajuda quando a doença já apresentou proporções substanciais. Há evidências de que esta realidade se deva à falta de conhecimento dos fatores de risco da doença e da relevância da detecção precoce para o prognóstico favorável. ${ }^{14-15}$ Além disso, reconhece-se, pelo menos em nosso país, que mulheres atendidas em serviços públicos apresentam pior prognóstico, o que tem sido particularmente atribuído ao fato de a doença ter sido diagnosticada em um estágio muito avançado. ${ }^{16}$

Diante do fato de que a falta de conhecimento sobre os riscos e as terapias pode levar a equívocos e à subutilização de prevenção e tratamento, justifica-se a importância deste estudo, sobre o conhecimento das mulheres acerca da doença e dos fatores de risco do câncer de mama, para subsidiar discussões que possam implementar programas e campanhas direcionadas a este público. ${ }^{17-19}$

Dessa forma, questiona-se: qual é conhecimento de mulheres sobre os fatores de risco para o câncer de mama? E para responder a esta questão o objetivo deste estudo foi descrever 


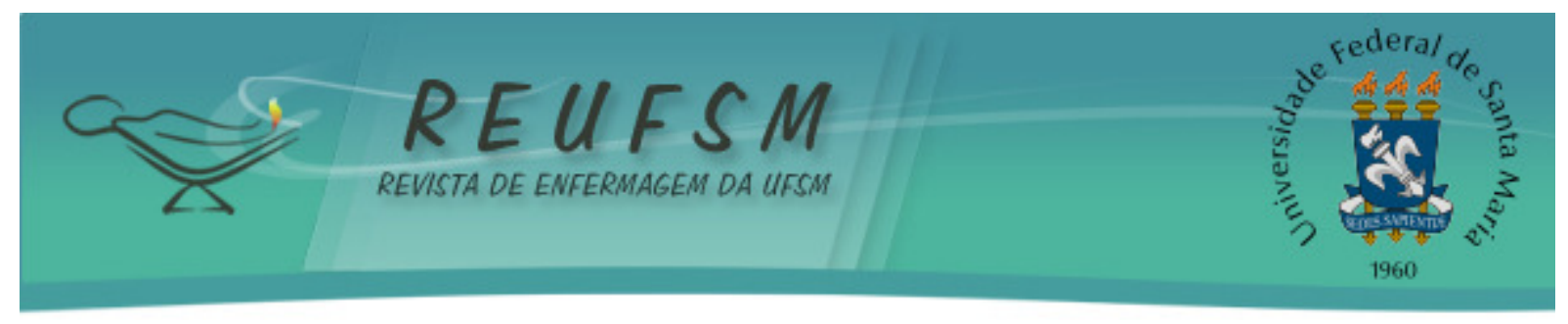

o perfil e o conhecimento de mulheres atendidas pela Estratégia Saúde da Família sobre os fatores relacionados ao câncer de mama.

\section{MÉTODO}

Trata-se de um estudo descritivo e transversal, com abordagem quantitativa, desenvolvido no município de Paranavaí, Paraná, no período de agosto de 2014 a outubro de 2015.

O público alvo foi constituído por mulheres cadastradas em 24 equipes da Estratégia Saúde da Família (ESF), alocadas em 12 Unidades Básicas de Saúde (UBS) com faixa etária de 35 a 69 anos. A população total correspondeu a 14.638 mulheres. Após cálculo de amostragem sistemática, por sorteio aleatório, com uma prevalência de câncer de mama de aproximadamente 80 por 100 mil, considerado o nível de confiança 95\% e erro tolerável de 4\%, obteve-se uma amostra mínima de 193 mulheres.

Após esclarecimentos em relação aos objetivos e método da pesquisa os dados foram coletados por meio de uma entrevista estruturada. ${ }^{20}$ Este instrumento foi composto por três blocos de perguntas. Tratou-se de um questionário estruturado de múltiplas escolhas, podendo ser assinaladas mais de uma opção. O primeiro bloco com identificação de variáveis sociodemográficas (idade, estado civil, escolaridade, etnia, religião, ocupação profissional), o segundo bloco com informações sobre a história reprodutiva (idade da menarca, idade da primeira relação sexual, número de filhos, idade materna no primeiro filho, tempo de amamentação, uso de anticoncepcionais orais) e o terceiro bloco abrangeu os conhecimentos relatados sobre os fatores de risco relacionados ao desenvolvimento do câncer de mama, no qual foi elencado, segundo os relatos das mulheres participantes: a história reprodutiva, uso de anticoncepcionais, estilo de vida, exposição à radiação, histórico familiar.

Foi realizado um teste piloto com 30 mulheres fora do plano amostral original com objetivo de verificar possíveis inconsistências e/ou dificuldades de interpretação das questões em relação ao objetivo da pesquisa. A organização, tabulação e sumarização dos dados foram realizadas no Microsoft Excel 2010 e apresentadas por meio de tabelas. Foram verificadas as medidas de proporções simples e medidas de tendência central por meio de estatística descritiva com o auxílio do software SAS (Statistical Analysis Software), versão 9.3.

A pesquisa foi submetida à análise das questões éticas de acordo com a Resolução 466/12 do Conselho Nacional de Saúde sendo aprovada em 09 de março de 2015 pelo Comitê 


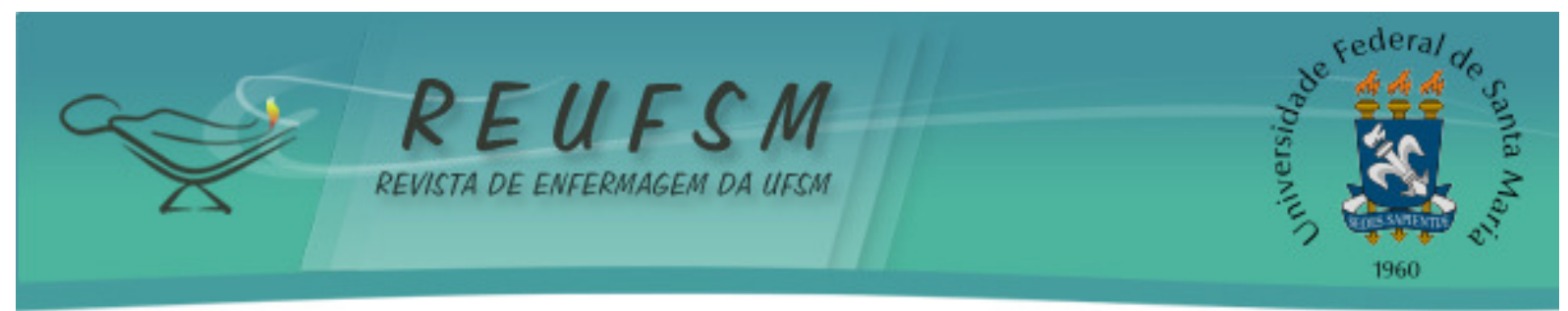

de Ética em Pesquisa com Seres Humanos do Centro Universitário Cesumar (UniCesumar) sob o parecer $\mathrm{n}^{\circ} 977.791$.

\section{RESULTADOS}

Entre as 193 participantes, a média de idade foi de 53 anos e faixa etária prevalente 50 a 60 anos (37,3\%); 64\% declararam-se casadas; 47\% sem estudo ou com o ensino fundamental incompleto; $67 \%$ católicas, $54 \%$ se declararam de etnia branca e $44 \%$ de etnia negro-parda. Notou-se que $31 \%$ das mulheres eram donas de casa e $24 \%$ aposentadas ou pensionistas e $63 \%$ declararam ter renda familiar mensal de um a três salários mínimos (Tabela 1).

Tabela 1 - Distribuição do perfil sociodemográfico das mulheres participantes do estudo na Estratégia Saúde da Família. Paranavaí, Paraná, 2014.

\begin{tabular}{|c|c|c|}
\hline Variáveis sociodemográficas & Frequência & $\%$ \\
\hline Faixa etária & & $\mathrm{NR}=0$ \\
\hline 35 I- 40 anos & 21 & 10,88 \\
\hline 40 I- 50 anos & 48 & 24,87 \\
\hline $50 \mid-60$ anos & 72 & 37,31 \\
\hline$\geq 69$ anos & 52 & 26,94 \\
\hline Estado civil & & $\mathrm{NR}=0$ \\
\hline Casada & 123 & 63,73 \\
\hline Separada & 17 & 8,81 \\
\hline Solteira & 28 & 14,51 \\
\hline Viúva & 25 & 12,95 \\
\hline Escolaridade & & $\mathrm{NR}=0$ \\
\hline Analfabetismo/ FI* & 91 & 47,15 \\
\hline $\mathrm{FC} / \mathrm{MI} *$ & 42 & 21,76 \\
\hline MC / SI & 46 & 23,83 \\
\hline SC / Especialização & 14 & 7,25 \\
\hline Religião & & $\mathrm{NR}=0$ \\
\hline Católico & 129 & 66,84 \\
\hline Evangélico & 57 & 29,53 \\
\hline Outras & 7 & 3,63 \\
\hline Etnia & & $\mathrm{NR}=0$ \\
\hline Branca & 105 & 54,40 \\
\hline Negra / parda & 86 & 44,56 \\
\hline Outras & 2 & 1,04 \\
\hline
\end{tabular}

Notas: NR: Não respondido;

*FI: Fundamental incompleto; FC: Fundamental completo; MI: Médio incompleto;

*MI: Médio incompleto; SI: Superior incompleto; SC: Superior completo. 


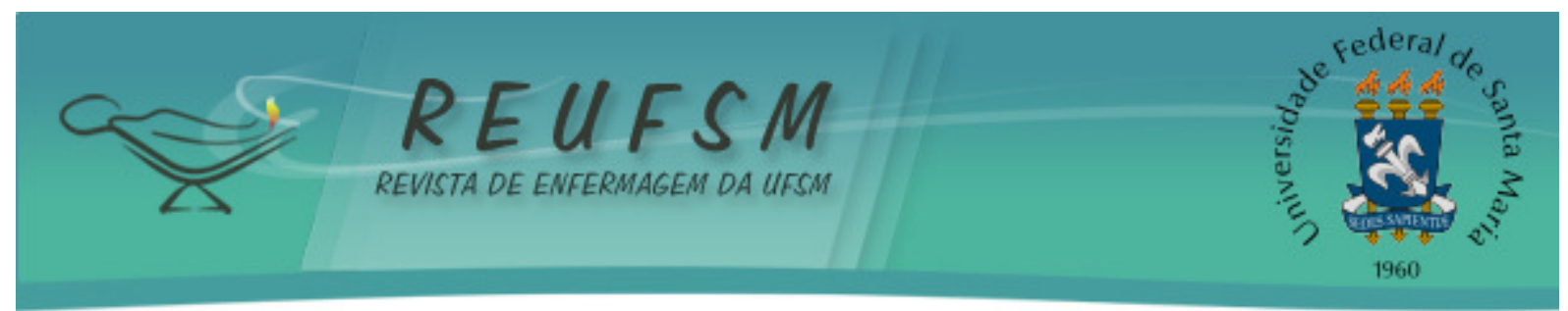

Com relação aos dados referentes à história reprodutiva, 58,73\% tiveram a menarca entre os 12 e os 14 anos de idade e 46,52\% tiveram a primeira relação sexual entre 16 e 20 anos de idade. Das entrevistadas, 91\% têm filhos, sendo que destas, 57\% gestaram com 20 a 29 anos de idade e $56 \%$ amamentaram por mais de seis meses. Nota-se também que $82 \%$ das entrevistadas já fizeram uso de anticoncepcionais e, dentre as que usaram, quase $40 \%$ usou por menos de cinco anos e $16 \%$ por 20 anos ou mais. Verificou-se que $38 \%$ das entrevistadas não se encontravam no climatério, enquanto que $27 \%$ já tinham iniciado com idade superior a 50 anos, sendo que $17 \%$ das mulheres fizeram uso de Terapia de Reposição Hormonal para o tratamento de sintomas da menopausa (Tabela 2).

Tabela 2 - Distribuição do perfil da história reprodutiva segundo relato das mulheres participantes do estudo na ESF. Paranavaí, Paraná, 2014.

\begin{tabular}{|c|c|c|}
\hline Variáveis da história reprodutiva & Frequência & $\%$ \\
\hline Idade da $1^{\circ}$ menstruação & & $\mathrm{NR}=4$ \\
\hline$<12$ anos & 34 & 17,99 \\
\hline $12 \mid-15$ anos & 111 & 58,73 \\
\hline$\geq 15$ anos & 44 & 23,28 \\
\hline Idade da $1^{\circ}$ relação sexual & & $\mathrm{NR}=6$ \\
\hline$<16$ anos & 21 & 11,23 \\
\hline $16 \mathrm{I}-20$ anos & 87 & 46,52 \\
\hline$\geq 20$ anos & 79 & 42,25 \\
\hline Filhos & & $\mathrm{NR}=0$ \\
\hline Não & 17 & 8,81 \\
\hline Sim & 176 & 91,19 \\
\hline Idade em que teve $1^{\circ}$ filho & & $\mathrm{NR}=17$ \\
\hline$<20$ anos & 64 & 36,36 \\
\hline 20 I- 30 anos & 101 & 57,39 \\
\hline$\geq 30$ anos & 11 & 6,25 \\
\hline Amamentou & & $\mathrm{NR}=13$ \\
\hline Sim,$<6$ meses & 55 & 30,56 \\
\hline Sim, $>6$ meses & 100 & 55,56 \\
\hline Não & 25 & 13,89 \\
\hline Anticoncepcional & & $\mathrm{NR}=0$ \\
\hline Não & 34 & 17,62 \\
\hline Sim & 159 & 82,38 \\
\hline
\end{tabular}

Nota: NR: Não respondido. 


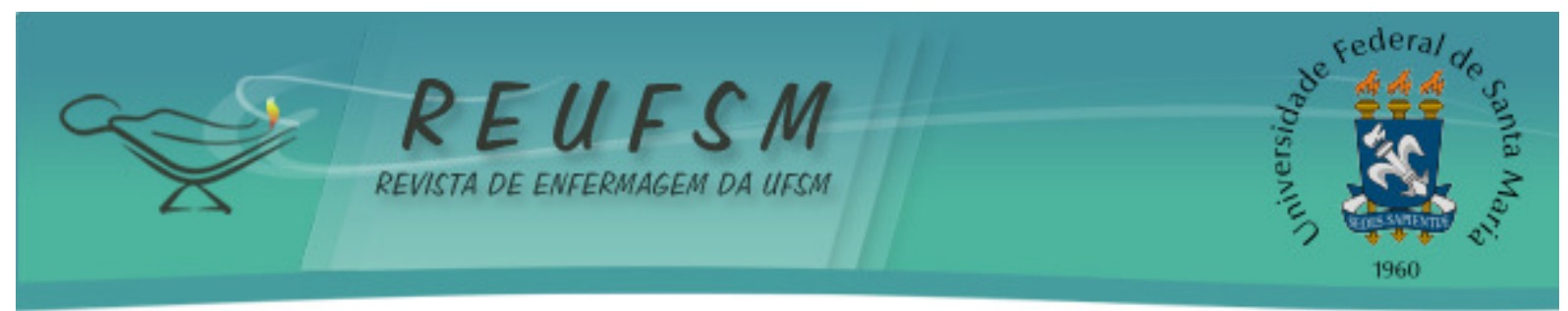

Quando questionadas acerca dos principais fatores relacionados ao aumento da incidência de câncer de mama, 22\% das respondentes apontaram as opções relacionadas ao histórico reprodutivo (idade, menstruação, gravidez, menopausa). O uso de anticoncepcionais orais foi citado por cerca de um quinto delas. Fatores relacionados ao histórico familiar foram citados por $52 \%$ das entrevistadas, embora $81 \%$ negaram história familiar de câncer de mama. Quanto aos fatores relacionados ao estilo de vida (obesidade, alcoolismo, tabagismo) foram assinalados por $42 \%$ das mulheres, ao passo que exposição a radiações por $13 \%$ delas. Outros fatores ainda foram citados por $55 \%$ das respondentes, sendo que os mais comuns foram alimentação, o fato de não amamentar e fatores psicológicos (Tabela 3).

Tabela 3 - Distribuição dos fatores de risco para desenvolvimento do câncer de mama, segundo relatos das mulheres participantes do estudo. Paranavaí, Paraná, 2014.

\begin{tabular}{l|cc}
\hline Fatores relacionados* & Frequência & \% \\
\hline & & $\mathrm{NR}=55$ \\
Histórico reprodutivo & 31 & 22,46 \\
Uso de anticoncepcionais orais & 28 & 20,29 \\
Estilo de vida & 58 & 42,03 \\
Exposição a radiações & 18 & 13,04 \\
Histórico familiar & 72 & 52,17 \\
Outro & 76 & 55,07 \\
\hline
\end{tabular}

Notas: NR: Não respondido;

$*$ mais de um fator poderia ser citado, sendo as frequências referentes a cada fator.

\section{DISCUSSÃO}

No presente estudo, com relação à raça/cor, as mulheres apresentaram proporções muito semelhantes entre si, no entanto, há evidências crescentes de que a mortalidade por câncer de mama é mais elevada em mulheres de ascendência africana do que européia. 3,21 Segundo um estudo realizado no Estado do Espírito Santo, mulheres classificadas com cor da pele preta apresentam 2,5 vezes mais risco de desenvolverem câncer de mama do que as de cor branca e, no mesmo estudo, o risco foi de 1,5 vezes maior quando comparadas com as mulheres de cor parda. ${ }^{22}$ Além disso, um estudo de coorte realizado em 239 hospitais do Brasil mostrou que mulheres com cor da pele diferente da branca apresentam maiores chances de atrasos no diagnóstico e tratamento do câncer de mama, sugerindo uma influência do fator étnico também no manejo do diagnóstico precoce. ${ }^{23}$ 


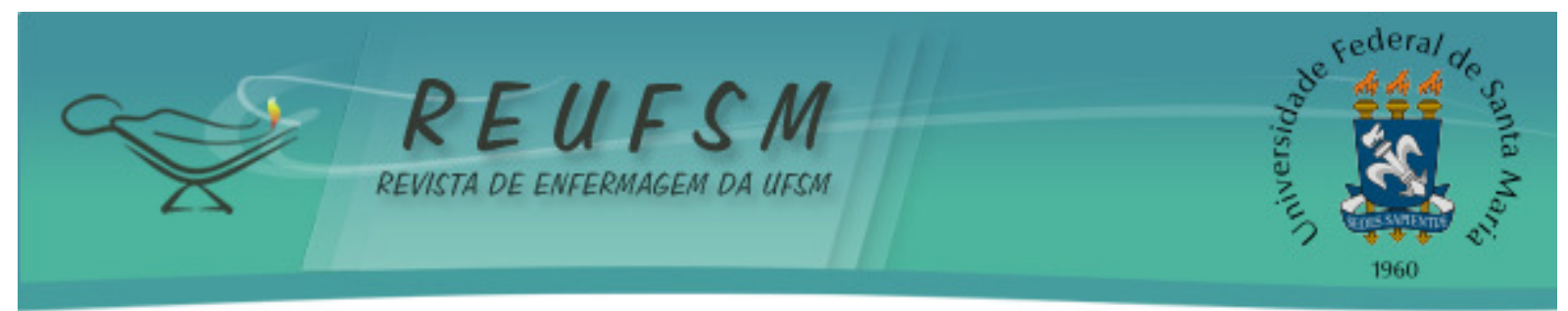

Outro ponto de destaque nesta pesquisa foi em relação à baixa escolaridade com altas proporções de analfabetismo, ou ensino fundamental incompleto. Uma análise dos determinantes que influenciam para início de tratamento para câncer de mama no Brasil, revelou que mulheres com menos de oito anos de escolaridade apresentavam mais riscos de atraso para o tratamento da doença. ${ }^{23}$ Uma investigação recente realizado no Estado de São Paulo, avaliou a implantação de um programa municipal de oferta de mamografias bianuais sem custos durante um período de dois anos, mostrou que aproximadamente $50 \%$ da população que nunca realizou mamografia se tratava de mulheres com baixo nível de instrução. ${ }^{14}$

Diante do fato de que o maior nível de escolaridade pode facilitar o acesso ao conhecimento e às informações, e que a população verificada apresenta nível de escolaridade baixo, é possível sugerir que este seja também um fator relevante a ser analisado para a detecção precoce do câncer de mama como também a ser incorporado nas elaborações de estratégias locais de melhora da adesão ao programa preventivo.

Ressalta-se que grande parte das mulheres entrevistadas utilizou anticoncepcional, sendo $20 \%$ destas por um período maior que 20 anos. As controvérsias relacionadas ao uso de anticoncepcionais e a neoplasia mamária são bastante encontradas na literatura especializada e entre os profissionais de saúde. O câncer de mama é uma doença do tipo hormônio sensível, em que o estrogênio tem sido implicado como principal agente cancerígeno. ${ }^{16}$ Por outro lado, a utilização de contraceptivos mistos, ou seja, aqueles que associam estrogênio e progestogênio parecem oferecer pouco risco ao desenvolvimento de neoplasias mamárias. ${ }^{7}$ Já $^{2}$ aqueles livres de estrogênio, ou seja, composto apenas por progestogênios, oferecem risco mínimo ou ausente de desenvolvimento de câncer de mama. ${ }^{16}$ Embora não fora avaliado a composição dos anticonceptivos utilizados, a constatação de que muitas mulheres fazem uso desta medicação por muitos anos, ressalta a necessidade de um melhor acompanhamento das mesmas pelas equipes da saúde da família.

Em relação aos fatores de risco relacionados com o aumento da incidência de câncer de mama, a história familiar foi citada de maneira prevalente como uma das principais causas para o câncer de mama. De fato, há estudos que mostram que 12-30\% dos cânceres de mama têm origem principalmente genética e são de herança autossômica dominante, resultado da mutação de vários genes, dentre os quais se destacam o BRCA1, BRCA2 e RAD50. 5,24 


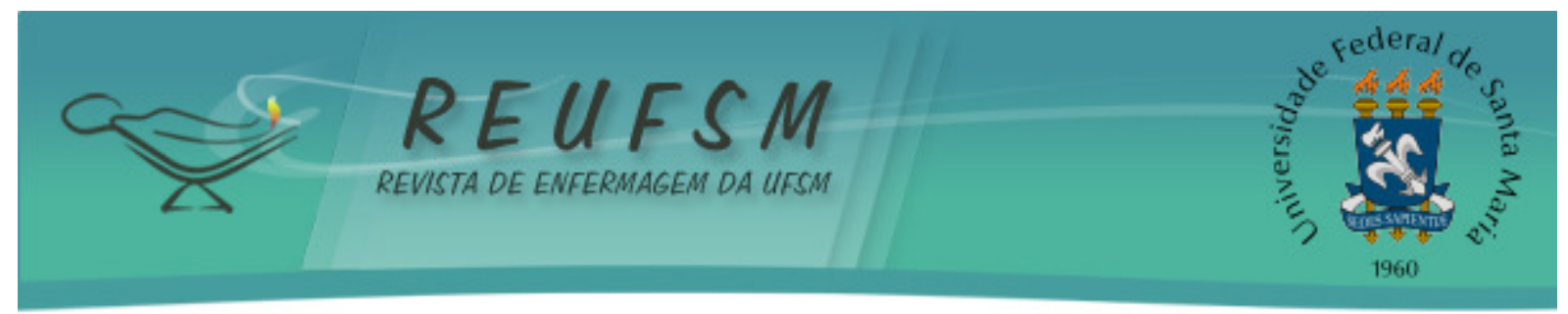

Apesar de haver o predomínio das mulheres participantes negarem casos de câncer de mama na família, o conhecimento sobre a importância deste fator de risco comprova que está sendo amplamente difundido. Além disso, fatores relacionados ao estilo de vida, em especial a obesidade, e o uso de álcool e tabaco foram também citados pela maioria das pesquisadas, mostrando que o conhecimento sobre os principais fatores de risco não parece ser um fator limitante para a adesão ao programa. No entanto, outros estudos devem ser realizados para verificar se a detenção destes conhecimentos faz, de fato, diferença no cotidiano destas mulheres e as mobilizem a aderirem ao programa preventivo.

Com relação à fonte de informação sobre câncer de mama, 76\% das mulheres afirmaram terem recebido informações por meio dos meios de comunicação. Embora não fora avaliado a eficácia das ações de mobilização dos profissionais da saúde envolvidos com o programa de prevenção do câncer de mama, os dados desta pesquisa apontam a necessidade de uma maior atuação destes profissionais na otimização da informação sobre a doença, a fim de garantir maior sensibilização da população alvo. Em estudo realizado em Londrina-PR, sobre o Programa de Controle e Prevenção do Câncer de Colo de Útero e Mama, verificou-se que as ações de prevenção para o câncer de mama pouco foram referidas pela população estudada. ${ }^{25}$

\section{CONCLUSÃO}

Traçar o perfil sociodemográfico e investigar o nível de conhecimento de mulheres cadastradas na Estratégia de Saúde da Família tornam-se indicadores imprescindíveis para desenvolvimento de ações de promoção e prevenção em saúde. Neste estudo as características sociodemográficas predominantes foram: mulheres casadas com 50 a 60 anos de idade, baixo nível de escolaridade, raça/cor branca e religião católica. Com relação ao conhecimento sobre os fatores de risco para câncer de mama, as mulheres participantes relataram aspectos concernentes com a literatura científica como história familiar, histórico reprodutivo e estilo de vida. Isto indica que o conhecimento pode ser satisfatório, porém as intervenções para redução de riscos necessitam ser incorporadas no cotidiano destas mulheres. Destaca-se o protagonismo dos profissionais de saúde na disseminação de informações e de práticas que minimizem os riscos de desenvolvimento do câncer de mama e de outros agravos crônico-degenerativos.

Como limitação, admite-se a possibilidade de viés de memória por se tratar de um estudo retrospectivo e o viés de prevaricação pela possibilidade de trazer algum 


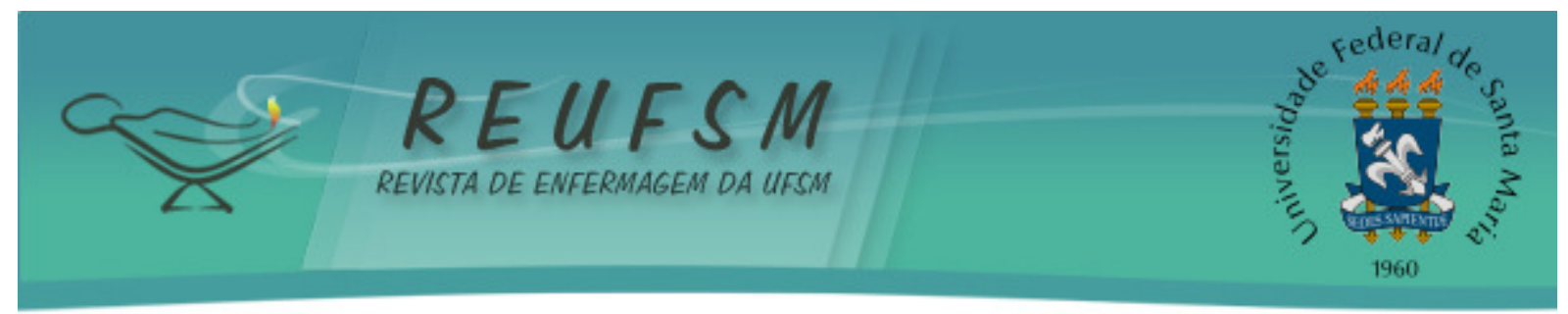

constrangimento a participante devido às perguntas relacionadas à sexualidade. A detecção precoce do câncer exige ações educativas permanentes, considerando que a atenção à saúde é permeada por um intenso e contínuo processo de produção e incorporação de inovações tecnológicas, todos direcionados em diminuir a morbidade e a mortalidade por câncer de mama.

\section{REFERÊNCIAS}

1. Ministério da Saúde. Instituto Nacional do Câncer José Alencar Gomes da Silva (Inca). Estimativa 2016: incidência de câncer no Brasil. Rio de Janeiro (RJ): INCA; 2016.

2. Pakzad R, Mohammadian-Hafshejani A, Khosravi B, SoltaniS, PakzadI, Mohammadian M, et al. The incidence and mortality of esophageal cancer and their relationship to development in Asia. Ann Transl Med [Internet]. 2016 jan [acesso em 2016 jan 17];4(2):29. Disponívelem:https://www.ncbi.nlm.nih.gov/pmc/articles/PMC4731602/.

3. DeSantis CE, Bray F, Ferlay J, Lortet-Tieulent J, Anderson BO, Jemal A. International variation in female breast cancer incidence and mortality rates. Cancer Epidemiol Biomarkers Prev [Internet]. 2015 out [acesso em 2016 jan 24];24(10):265464. Disponível em: https://www.ncbi.nlm.nih.gov/pubmed/26359465.

4. TorreLA,Siegel RL, Ward EM, Jemal A. Global cancer incidence and mortality rates and trends-an update. Cancer Epidemiol Biomarkers Prev [Internet]. 2016 jan [acesso em 2016 fev 10];25(1):16-27. Disponível em: https://www.ncbi.nlm.nih.gov/pubmed/26667886.

5. Kaplan HG, Malmgren JA, Atwood MK, Calip GS. Effect of treatment and mammography detection on breast cancer survival over time: 1990-2007. Cancer [Internet]. 2015 ago [acesso em 2016 jan 18];121(15):2553-61. Disponível em: https://www.ncbi.nlm.nih.gov/pubmed/25872471.

6. Løberg M, Lousdal ML, BretthauerM, Kalager M. Benefits and harms of mammography screening. Breast Cancer Res [Internet]. 2015 maio [acesso em 2016 jan 23];17(1):63. Disponível em: https://www.ncbi.nlm.nih.gov/pmc/articles/PMC4415291/.

7. Alves MO, Magalhães SCM, Coelho BA. Contribuições da geografia médica para o estudo do câncer de mama. Hygeia (Uberlândia).2014;10(19):86-96.

8. Tatalovich Z, Zhu L, Rolin A, Lewis DR, Harlan LC, Winn DM. Geographic disparities in late stage breast cancer incidence: results from eight states in the United States. Int J Health Geogr [Internet]. 2015 oct [acesso em 2016 jan24];14(31).Disponível em: https://www.ncbi.nlm.nih.gov/pmc/articles/PMC4619382/.

9. Farolfi A, Scarpi E, RoccaA,Mangia A, Biglia N, Gianni L, et al. Time to initiation of adjuvant chemotherapy in patients with rapidly proliferating early breast cancer.EurJCancer (1990) [Internet]. 2015 set [acesso em 2016 fev 13];51(14):1874-81.Disponível em: https://www.ncbi.nlm.nih.gov/pubmed/26206258.

10. Cecilio AP, Takakura ET, JumesJJ, Santos JW, Herrera AC, Victorino VJ, et al. Breast cancer in Brazil: epidemiology and treatment challenges. Breast Cancer (Dove Med Press) 


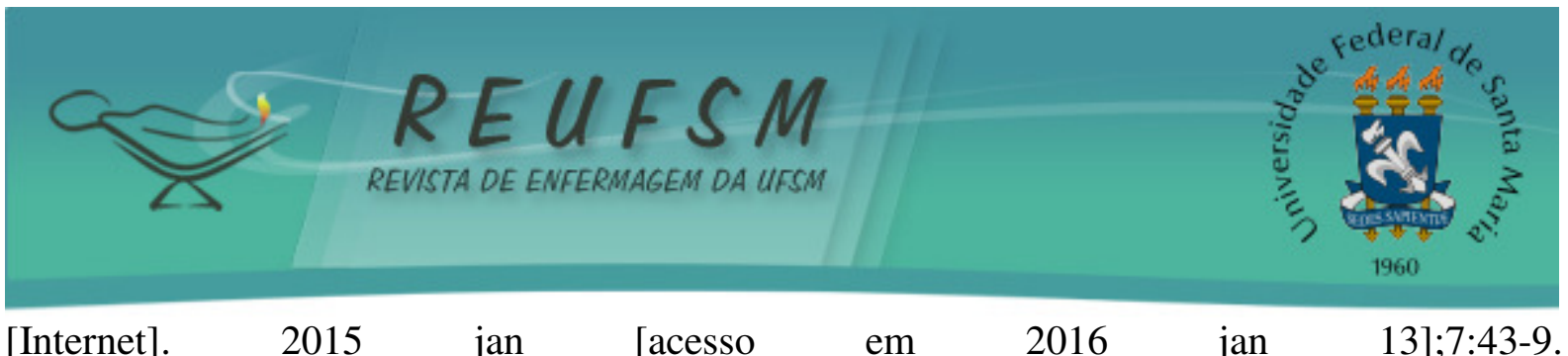

$\begin{array}{lllllll}\text { [Internet]. } & 2015 & \text { jan } & \text { [acesso em } & 2016 & \text { jan } & \text { 13];7:43-9. }\end{array}$ Disponívelem:https://www.ncbi.nlm.nih.gov/pmc/articles/PMC4317062/.

11. Kluthcovsky ACGC, Faria TNP, Carneiro FH, Strona R. Female breast cancer mortality in Brazil and its regions. Rev Assoc Med Bras [Internet]. 2014 set [acesso em 2016 jan 23];60(4):387-93. Disponível em: http://www.scielo.br/pdf/ramb/v60n4/0104-4230-ramb-6004-0387.pdf.

12. Gonzaga CMR, Freitas Junior R, Curado MP, Sousa ALL, Souza Neto JÁ, Souza MR. Temporal trends in femalebreastcancer mortality in Brazil and correlations with social inequalities: ecological time-series study. BMC Public Health [Internet]. 2015 fev [acesso em 2016 fev 24];7(1):15-96. Disponível em: http://bmcpublichealth.biomedcentral.com/articles/10.1186/s12889-015-1445-7.

13. Global Burden Of Disease Cancer Collaboration, Fitzmaurice C, Dicker D, Pain A, Hamavid H, Moradi-Lakeh M, et al. The Global Burden of Cancer 2013. JAMA Oncology [Internet]. 2015 jul [acesso em 2016 jan 20];1(4):505-27. Disponível em: https://www.ncbi.nlm.nih.gov/pubmed/26181261.

14. Vieira RAC, Lourenço T, Mauad EC, Moreira Filho VG, Peres SV, Silva TB, et al. Barriers related to non-adherence in a mammography breast-screening program during the implementation period in the interior of São Paulo State, Brazil. J Epidemiol Glob Health [Internet]. 2015 set [acesso em 2016 fev 17];5(3):211-9. Disponível em: https://www.ncbi.nlm.nih.gov/pubmed/26231397.

15. Guerra MR, Silva GA, Nogueira MC, Leite ICG, Oliveira RVC, Cintra JRD, et al . Sobrevida por câncer de mama e iniquidade em saúde. Cad Saúde Pública [Internet]. 2015 ago [acesso em 2016 abr24];31(8):1673-84. Disponível em: http://www.scielo.br/pdf/csp/v31n8/0102-311X-csp-31-8-1673.pdf.

16. Wells AA, Shon EJ, McGowan K, James A. Perspectives of low-income africanamerican women non-adherent to mammography screening: the importance of information, behavioral skills, and motivation. J Cancer Educ [Internet]. 2015 dez [acesso em 2016 jan 20];32(2):328-34. Disponível em: https://www.ncbi.nlm.nih.gov/pubmed/26661256.

17. Livaudais-Toman J, Karliner LS, Tice JA, Kerlikowske K, Gregorich S, Pérez-Stable EJ, et al. Impact of a primary care based intervention on breast cancer knowledge, risk perception and concern: a randomized, controlled trial. Breast [Internet]. $2015 \mathrm{dez}$ [acesso em 2016 jan 18];24(6):758-66. Disponível em: https://www.ncbi.nlm.nih.gov/pubmed/26476466.

18. Damiani G, Basso D, Acampora A, Bianchi CB, Silvestrini G, FrisicaleEM, et al. The impact of level of education on adherence to breast and cervical cancer screening: Evidence from a systematic review and meta-analysis. Prev Med[Internet]. $2015 \mathrm{dez}$ [acesso em 2016 fev 22];81:281-9. Disponível em: https://www.ncbi.nlm.nih.gov/pubmed/26408405.

19. Costa AR, Silva S, Moura Ferreira P, Villaverde Cabral M, Santos O, Carmo I, et al. Health-related knowledge of primary prevention of cancer in Portugal. Eur J Cancer Prev [Internet]. 2016 jan [acesso em 2016 mar 25];25(1):50-3. Disponível em: https://www.ncbi.nlm.nih.gov/pubmed/25642793.

20. Batiston AP. Detecção precoce do câncer de mama: conhecimento e prática de mulheres e profissionais da Estratégia de Saúde da Família em Dourados/MS. Rev Bras Ginecol Obstet 


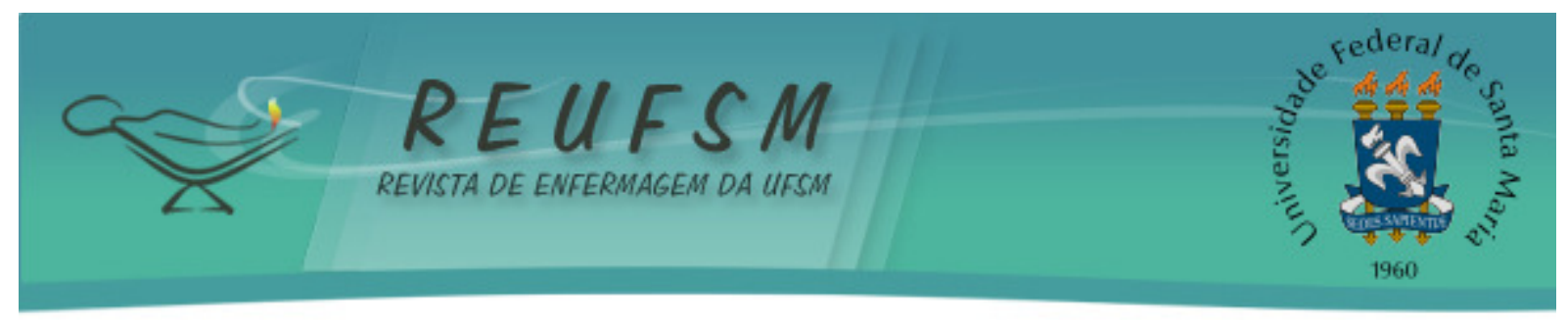

[Internet]. 2010 fev [acesso em 2016 abr 24];32(2):90-9. Disponível em: http://www.scielo.br/scielo.php?script=sci_arttext\&pid=S0100-72032010000200010.

21. Brewster AM, Chavez-Macgrego ME, Brown P. Epidemiology, biology, and treatment of triple-negative breast cancer in women of African ancestry. Lancet Oncol [Internet]. $2014 \mathrm{dez}$ [acesso em 2016 fev 22];15(13):625-34. Disponível em: https://www.ncbi.nlm.nih.gov/pubmed/25456381.

22. Silva PF,Amorim MHC, ZandonadeE,Viana KCG. Associação entre variáveis sociodemográficas e estadiamento clínico avançado das neoplasias da mama em Hospital de Referência no Estado do Espírito Santo. Rev Bras Cancerol. 2013;59(3):361-7.

23. Medeiros GC, Bergmann A, Aguiar SS, Thuler LCS. Análise dos determinantes que influenciam o tempo para o início do tratamento de mulheres com câncer de mama no Brasil. Cad Saúde Pública [Internet]. 2015 jun [acesso em 2016 abr 24];31(6):1269-1282. Disponível em: http://www.scielo.br/pdf/csp/v31n6/0102-311X-csp-31-6-1269.pdf.

24. Lin PH, Kuo WH, Huang AC, Lu YS, Lin CH, Kuo SH, et al. Multiple gene sequencing for risk assessment in patients with early-onset or familial breast cancer. Oncotarget [Internet]. 2016 fev [acesso em 2016 fev 22];7(7): 8310-20. Disponível em: https://www.ncbi.nlm.nih.gov/pubmed/26824983.

25. Pinho MCV, Jodas DA, Scochi MJ.Profissionais de saúde e o programa de controle do câncer do colo uterino e mama. Rev Enferm UFSM [Internet]. 2012 maio/ago;2(2):242-51. Disponível em: https://periodicos.ufsm.br/reufsm/article/view/4418/3748.

Data de submissão: 18/01/2017

Data de aceite: $21 / 09 / 2017$

Autor principal: Neide Derenzo.

Endereço: Rua Padre Antonio Vieira, 680-Bairro São Jorge, Paranavaí, Paraná, Brasil

CEP: 87710-030

Email: neidederenzo@hotmail.com 